\title{
Influencia en la autoeficacia del grado de autenticidad de la indagación de dos proyectos de ciencia de secundaria. Estudio de caso
}

\author{
Carme Grimalt-Álvaro \\ Departament Pedagogia, Facultat de Ciències de l'Educació i Psicologia, Universitat Rovira i Virgili. \\ Tarragona.carme.grimalt@urv.cat \\ Enric Ortega-Torres \\ Unitat d'Educació, Florida Universitaria i Departament de Didàctica de les ciències experimentals, Facultat \\ de Magisteri, Universitat de València, València eortega@florida-uni.es
}

Digna Couso

Centre per a l'Educació Científica i Matemàtica (CRECIM) i Departament de Didàctica de les Matemàtiques i les Ciències Experimentals, Facultat de Ciències de l'Educació, Universitat Autònoma de Barcelona,Bellaterradigna.couso@uab.cat

Laura Paloma Romeu

Institut Cardener. Sant Joan de Vilatorrada.Ipaloma@xtec.cat

[Recibido: 17 junio 2020. Revisado: 30 junio 2020. Aceptado: 23 octubre 2020]

\begin{abstract}
Resumen: Las creencias sobre la propia capacidad para llevar a cabo con éxito una tarea concreta (autoeficacia) influyen en la implicación y la motivación hacia dicha tarea. En las últimas décadas se ha incrementado el foco de atención hacia estas creencias en el área de ciencias no sólo como uno de los predictores más consistentes del desempeño real del alumnado, sino por la existencia de desigualdades sistemáticas en la autoeficacia de chicos y chicas. Aunque el Aprendizaje Basado en Proyectos (ABP) en el aula de ciencias posee diversas características que potencialmente pueden contribuir a la mejoría de la autoeficacia en ciencias del alumnado, las evidencias entre la relación de los elementos de diseño y la mejora de la autoeficacia en ciencias del alumnado han sido poco exploradas. Con la finalidad de ofrecer pautas para el diseño, la investigación que se presenta pretende explorar qué características de la indagación planteada en dos variaciones del proyecto "El congreso científico" pueden promover la mejora en la autoeficacia en las ciencias de los y las estudiantes de 14-15 años participantes en dos centros de secundaria (A y B). Se diseñó un cuestionario que respondieron 60 estudiantes antes y después de su participación en el proyecto (10 chicos y 16 chicas en el Centro A y 23 chicos y 11 chicas en el Centro B). Los resultados muestran que estrategias de indagación que otorgan diferentes grados de autenticidad en la indagación científica escolar no derivan necesariamente en un aumento de la autoeficacia en ciencias a corto plazo. En el artículo se discute la necesidad de incluir otras estrategias complementarias para garantizar que tanto chicas como chicos pueden tener experiencias de indagación científica con éxito real.
\end{abstract}

Palabras clave: autoeficacia, aprendizaje basada en proyectos, educación secundaria, indagación

Influence on self-efficacy of the degree of authenticity of the inquiry carried out in two secondary school science projects

Abstract: Beliefs about one's ability to successfully carry out a specific task (self-efficacy) influence involvement and motivation towards that task. In recent decades, the focus of attention on these beliefs has increased in the area of science education, not only as one of the most consistent predictors of students' real performance, but because of the existence of systematic inequalities regarding boys' and girls' self-efficacy. Although various characteristics of Project Based Learning (PBL) can potentially contribute to the improvement of students' selfefficacy, the evidence on the relationship between the elements of design and the improvement of students' selfefficacy in school science has been little explored. To offer design guidelines, the research presented aims at 
exploring which characteristics of the inquiry carried out in two variations of "The scientific congress" project can lead to an improvement in self-efficacy of 14-15-year-old participating students in two secondary schools (A and B). A questionnaire was designed and answered by 60 students before and after their participation in the project (10 boys and 16 girls from School A, and 23 boys and 11 girls from School B). Results show that research strategies with different degrees of authenticity in scientific inquiry in school do not necessarily lead to an improvement in science self-efficacy of students in the short term. The article discusses the need to include other complementary strategies to guarantee that both girls and boys can successfully participate in real scientific research experiences.

Keywords: self-efficacy, Project-Based Learning, secondary education, inquiry

Para citar este artículo: Grimalt-Álvaro C., Ortega-Torres E., Couso D. y Paloma Romeu L. (2021) Influencia en la autoeficacia del grado de autenticidad de la indagación de dos proyectos de ciencia de secundaria. Estudio de caso. Revista Eureka sobre Enseñanza y Divulgación de las Ciencias 18(2), 2101. doi: 10.25267/Rev_Eureka_ensen_divulg_cienc.2021.v18.i2.2101

\section{Introducción}

Antes de llevar a cabo una tarea, por pequeña que sea, cualquier persona tendrá una intuición acerca de si será capaz, o no, de llevarla a cabo. En la mayoría de las acciones cotidianas el grado de convencimiento de éxito será absoluto. En cambio, para acciones que suponen un mayor reto, la persona seguramente tomará un tiempo mayor para evaluar sus propias competencias y decidir en qué medida cree que será capaz de llevar a cabo dicha tarea con éxito. El conjunto de creencias en las propias capacidades para organizar y ejecutar las diversas acciones necesarias para gestionar con éxito una situación futura se engloba dentro del término autoeficacia definido por Bandura (1997). Estas creencias se encuentran intrínsecamente vinculadas a la acción determinada que se pretende realizar -lo que explica por qué una persona pueda sentirse capaz de cocinar una tortilla, pero no de arreglar una cisterna que pierde agua- y para un nivel concreto de dificultad (Bandura, 2012) -una persona podrá sentirse capaz de cocinar una tortilla para 2 personas, pero no para 100 comensales.

Dada su relación con una acción futura, la autoeficacia se considera un componente de la motivación, de acuerdo con los modelos cognitivo-sociales (Linnenbrink y Pintrich, 2002). Estos modelos enfatizan que el alumnado puede motivarse de diversas maneras y que la motivación no es un rasgo estable de un individuo, sino que depende del contexto y es específica del dominio (Linnenbrink y Pintrich, 2002). En particular, la autoeficacia se ha estudiado ampliamente en el ámbito educativo, puesto que la manera en que el alumnado interpreta los resultados de sus propias acciones informa y altera las acciones futuras (Pajares, 2006). Esto es particularmente crítico en los niveles de secundaria y universidad, donde los y las estudiantes tienen cada vez más opciones académicas disponibles para ellos (Pajares, 2006).

Partiendo de este marco, Britner y Pajares (2006) definieron la autoeficacia en ciencias como el conjunto de las creencias del alumnado acerca de sus habilidades para tener éxito en las actividades del ámbito científico. En las últimas décadas se ha incrementado el foco de atención hacia este constructo porque se ha evidenciado el rol de la autoeficacia en ciencias como uno de los predictores más consistentes del desempeño real del alumnado: alumnado con una autoeficacia alta muestra un desempeño alto en las asignaturas relacionadas, y al revés (Usher, 2009). Además, también se ha demostrado que el alumnado con niveles altos de autoeficacia en ciencias tiende a implicarse más en las actividades, lo que también repercute en el desempeño final obtenido. Esta relación se explica desde la influencia de la autoeficacia en cómo piensa y siente cada persona acerca de sí misma, sus propias expectativas de éxito, la motivación que comportan y, consecuentemente, los actos que se derivan de esta motivación (Bandura, 1997). Promover un nivel adecuado de autoeficacia en ciencias en el alumnado no sólo es necesario para un buen desarrollo de la alfabetización científica, sino para garantizar 
una actitud positiva hacia el ámbito y las actividades científicas en las que pueda verse involucrado en un futuro como ciudadano, independientemente de sus elecciones profesionales.

Desafortunadamente, diversas investigaciones suministrados o datos empíricos no generaría desigualdad entre el alumnado. En la misma línea, otro eje habitual de caracterización del grado de autenticidad enmuestran la existencia de desigualdades sistemáticas en la autoeficacia en ciencias según el género. Para un mismo nivel de desempeño, las chicas tienden sistemáticamente a subestimar sus capacidades en el área científico-tecnológica, mientras que los chicos tienden a sobrevalorarlas (Baker y White, 2003; Britner, 2008; Huang, 2013; WebbWilliams, 2018). Informes a nivel mundial, como los resultados de las pruebas PISA muestran cómo estas desigualdades son una constante en la mayoría de los países y, curiosamente, son más pronunciadas en aquellos países con mayor igualdad de género a nivel social (Mostafa, 2019). Las intersecciones entre el género y la pertenencia a minorías étnicas, y/o nivel socioeconómico (p.ej. una chica latina, respecto a sus compañeros) amplificarían aún más este efecto negativo en la autoeficacia (Ro y Loya, 2015). El problema de esta diferente autoeficacia debe entenderse en relación con el estereotipo social de que la ciencia -y en particular, algunas disciplinas como la física- se concibe como algo difícil y complejo, para lo que es necesario ser una persona extraordinariamente brillante y muy inteligente (Archer et al., 2013). Esta visión estereotipada aleja aún más aquellas personas que no sienten que comparten estas mismas características, reforzando que las chicas no desarrollen una actitud positiva hacia este ámbito.

\section{Promover la autoeficacia en ciencias desde el Aprendizaje Basado en Proyectos}

Bandura (1997) definió cuatro grandes ámbitos de influencia a partir de los cuales se desarrolla positivamente la autoeficacia, siendo la experiencia de éxito genuino el más efectivo entre ellos. La concreción de estas fuentes en estrategias educativas a nivel general ha sido estudiada en algunas publicaciones. Por ejemplo, Bandura (1997) relaciona cómo llevar a cabo actividades que faciliten que el alumnado pueda definir y seguir objetivos de logro sucesivos y adecuadamente escalonados puede ser positivo para la construcción de la autoeficacia, puesto que promueve las experiencias de éxito. Así, actividades donde se promueven estrategias de autorregulación y autoevaluación del aprendizaje en el alumnado tienen un impacto positivo en la autoeficacia del alumnado, aunque el impacto es más elevado en aquellos/as estudiantes con un mayor nivel de autoeficacia (Lai, Hwang y Tu, 2018). Desde esta misma perspectiva, Bandura (1997) también recomienda el diseño de secuencias de enseñanza - aprendizaje que ofrezcan diversos itinerarios posibles para alcanzar los objetivos de aprendizaje, en comparación a secuencias cerradas con un único camino. Actividades en las que se promueve un trabajo cooperativo entre estudiantes contribuyen positivamente a incrementar los niveles de autoeficacia del alumnado a través de las experiencias de éxito indirecto con el modelo social que representan los/as compañeros/as (Fernandez, 2017; Webb-Williams, 2018).

El Aprendizaje Basado en Proyectos (ABP) en el aula de ciencias posee diversas características que potencialmente pueden contribuir a la mejoría de la autoeficacia en ciencias del alumnado a través de alguna de las fuentes o estrategias descritas anteriormente. Una de las características que más se valora en el enfoque ABP en la enseñanza y aprendizaje de las ciencias es el grado de autenticidad de la indagación científica (Hasni, 2016), que también tiene relación con la promoción de la autoeficacia en el alumnado (Baker y White, 2003; Beier et al., 2019). El grado de autenticidad está relacionado con el grado de participación activa del alumnado en la selección de la demanda, los contenidos que se desarrollan el proyecto, el contexto, así como el tipo de prácticas de indagación, entre otros aspectos (Thomas, 2000).

En relación con la autenticidad en el tipo de prácticas indagativas, se identifica en la literatura un continuo que se engloba entre dos extremos: en uno se encuentran aquellos proyectos en 
los que los datos recopilados y tratados son totalmente hipotéticos y suministrados, es decir, datos obtenidos por terceras personas o mediante el uso de fuentes en la literatura (JiménezLiso, 2020). En otro extremo, se sitúan aquellos proyectos de indagación en los que los datos usados para la obtención de pruebas son empíricos y obtenidos directamente por el propio alumnado (Jiménez-Liso, 2020). Aunque desde otras metodologías educativas similares, como el aprendizaje basado en problemas, se ha reportado que el alumnado con menor nivel de autoeficacia tiene más dificultades inicialmente en la toma de datos con respecto al alumnado con mayor nivel de autoeficacia (Ketelhut, 2007), estas diferencias desaparecerían a medida que ambos grupos de estudiantes participasen en la actividad o secuencia de enseñanza aprendizaje (Ketelhut, 2007). Así pues, desde este marco, el uso de datos hipotéticos y suministrados o datos empíricos no generaría desigualdad entre el alumnado.

En la misma línea, otro eje habitual de caracterización del grado de autenticidad en las prácticas de indagación se encuentra en el grado de apertura de dichas prácticas, pudiéndose encontrar desde proyectos completamente guiados por el profesorado, a proyectos totalmente abiertos en que el alumnado decide la temática del proyecto y el diseño experimental (Domènech-Casal, 2018).

Aunque estos diferentes planteamientos en las prácticas de indagación de los proyectos de ciencias puedan conferir un nivel de autenticidad distinto, no existen en la literatura referencias de la relación de estos elementos de diseño en el ABP con la promoción de la autoeficacia en ciencias del alumnado (Beier et al., 2019; Rittmayer y Beier, 2009). Así pues, con la finalidad de ofrecer pautas para el diseño de proyectos que promuevan la autoeficacia del alumnado en ciencias, la investigación que se presenta pretende explorar cómo el grado de autenticidad de un proyecto de ciencias pueden promover la mejora en la autoeficacia en las ciencias de los y las estudiantes de secundaria participantes según su género.

\section{Metodología}

La investigación se llevó a cabo en dos centros educativos de secundaria (Centro A y Centro B) vinculados a una red de institutos innovadores vinculada a la Universitat Autònoma de Barcelona. Del conjunto de 9 centros que formaban la red, el Centro A y el Centro B se ofrecieron voluntarios para participar en el proyecto europeo STEAM4U (2016-1-ES01KA201-025633) que tenía como objetivo a aumentar la autoeficacia del alumnado en el ámbito STEM (ciencias, tecnología, ingeniería y matemáticas, por sus siglas en inglés) mediante la implementación y análisis de diversas estrategias educativas. En particular, estos dos centros buscaron aumentar la autoeficacia del alumnado de 14-15 años ( $3^{\circ}$ de ESO) realizando propuestas diferenciadas de un mismo proyecto de indagación científica llamado "El congreso científico". Las dos versiones del proyecto surgieron a partir de la revisión de una propuesta inicial en una reunión conjunta con los equipos docentes de ambos centros y fueron refinadas por cada equipo en su centro posteriormente. La implementación de ambos proyectos se llevó a cabo en el curso 2017-2018.

En esta investigación nos preguntamos cómo estas dos versiones del proyecto, que poseen elementos de diseño diferenciados en relación con el grado de autenticidad de la indagación científica en el aula, pueden afectar a la autoeficacia en ciencias del alumnado participante a corto plazo según su género. La investigación se realiza desde un paradigma interpretativo en el que se hace uso de una metodología cuantitativa para dar respuesta al objetivo. Así, el presente trabajo no se plantea como una comparación entre dos centros, sino como dos estudios de caso relacionados, siguiendo a Harrison, et al. (2017).

A continuación, se describen el contexto de los dos centros, así como los elementos de diseño diferenciados en los dos proyectos implementados. 


\section{“El congreso científico" en el Centro A}

El Centro A es un centro donde se imparten exclusivamente estudios de secundaria y bachillerato. Una de las características peculiares del centro es su organización como cooperativa. Este funcionamiento colaborativo se traslada al trabajo con el alumnado, siendo la metodología ABP habitual. Las familias del alumnado tienen un nivel sociocultural medioalto según la referencia del índice ISEC (Índice socioeconómico cultural). El porcentaje de alumnado inmigrante es bajo $(<5 \%)$ así como el porcentaje de alumnado con necesidades educativas especiales.

En el Centro A, el reto planteado al alumnado consistió en realizar una investigación bibliográfica sobre un tema científico propuesto por el profesorado con la finalidad de defender las conclusiones obtenidas frente a docentes universitarios expertos en la materia investigada. Los temas se presentaban mediante preguntas que pudieran generar curiosidad en el alumnado (Figura 1). El alumnado seleccionaba de manera individual sus preferencias y, a partir de éstas, se agrupaba y se configuraron grupos heterogéneos de trabajo de 4 estudiantes, con roles definidos para promover y facilitar la participación de todos los miembros. Los grupos de trabajo tenían la posibilidad de ampliar y modificar la pregunta inicial de acuerdo con los propios intereses del grupo y/o los resultados obtenidos a medida que avanzaba la búsqueda bibliográfica y el análisis de las diversas fuentes obtenidas. De esta manera, se pretendía acercar la investigación a las preocupaciones reales y necesidades de cada equipo de trabajo.
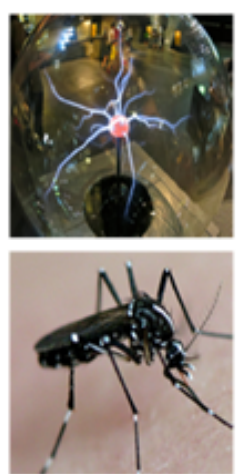

\section{TEMA 2: LA ELECTRICIDAD Y SU PROCEDENCIA}

Cada vez más podemos ver cómo van apareciendo en el mercado coches eléctricos. ¿De dónde sacan la electricidad estos coches para funcionar? ¿De dónde proviene la electricidad que utilizamos en nuestra casa? ¿Sería mejor si todos los vehículos fueran eléctricos? ¿Cuál es la mejor forma de obtener electricidad?

\section{TEMA 3: EL VIRUS Zika}

El virus Zika ha afectado a personas en más de 30 países. ¿Qué es un virus? ¿Cuáles son los efectos de este virus? ¿A qué órganos o tejidos afecta? ¿Cómo se transmite? ¿Hay tratamiento? ¿Se podría curar con antibióticos? ¿Qué es un suero? ¿Y una vacuna?

Figura 1. Ejemplo de pregunta investigable para la elección del alumnado en el Centro A.

Para facilitar el acompañamiento del alumnado, el profesorado del Centro A diseñó un proceso de trabajo donde existían entregas parciales de diferentes productos de investigación a lo largo de todo el proyecto. Esta necesidad de finalizar pequeños productos y recibir respuesta por parte del equipo de docentes en reuniones semanales (llamadas sesiones de parada) mantenía el proceso de búsqueda activo y permitía ampliar la profundidad de la investigación a lo largo de los dos meses de duración del proyecto. Además de cada entregable, se diseñaron y proporcionaron previamente una base de orientación y una rúbrica de evaluación específica al alumnado. La base de orientación incluía las características esenciales que debía de cumplir cada entregable: extensión, estructura y apartados con la descripción del contenido esperado. La rúbrica contenía los criterios de evaluación y los niveles de logro con que posteriormente se evaluaría el producto final entregado. El objetivo del profesorado fue favorecer la regulación del propio aprendizaje a lo largo del desarrollo del proyecto, proporcionando la información necesaria para que el estudiante supiese hacia dónde debía enfocar su avance, conocer el punto del camino en que se encontraba y saber qué había aprendido hasta el momento y aquello que le quedaba por aprender. La defensa de la investigación bibliográfica realizada frente a un 
comité de expertos externos al centro fue el momento culmen del proyecto en el Centro A. Para ayudar a la preparación de este evento, se realizaron ensayos previos de cada grupo de trabajo delante del grupo clase. Para los grupos de trabajo, esta última parte supuso un extra de motivación y de 'estrés' simultáneamente.

\section{“El congreso científico" en el Centro B}

El Centro B es un instituto público donde se imparte exclusivamente estudios de secundaria obligatoria (ESO). El centro ha reorganizado toda la distribución horaria, planteando el trabajo del conjunto de materias en cinco áreas de conocimiento donde la presencia de la metodología ABP toma una especial relevancia. Las familias del alumnado tienen un nivel sociocultural medio y un perfil homogéneo, puesto que la proporción de estudiantes de origen inmigrante no llega al 1\%. La proporción de alumnado con necesidades específicas de apoyo educativo es alta en el centro, alrededor del 13\%.

El reto planteado al alumnado del Centro B consistió en realizar una investigación científica de carácter empírico por parejas sobre un tema o pregunta planteada por el propio alumnado, con la finalidad de llevar a cabo un intercambio de conocimiento final emulando un congreso científico. Así pues, el alumnado fue el responsable de concretar qué tema o pregunta quería investigar de acuerdo con sus propios intereses, y las preguntas fueron refinadas con la ayuda del profesorado. La propuesta de preguntas y refinamiento se hizo después de diversas actividades basadas en la actividad clásica de las mystery boxes (Michaels et al., 2007), donde el alumnado pudo discutir las diversas prácticas científicas y clarificar las etapas de una indagación. Una vez refinada la pregunta de investigación, las parejas de trabajo diseñaron un experimento para tomar datos y responder su pregunta, plantear una predicción o hipótesis, interpretar los datos obtenidos, y extraer conclusiones.

Durante todas las etapas de la indagación, el profesorado promovía la reflexión del alumnado en los encuentros informales en las sesiones de trabajo presencial. Al final de cada sesión cada estudiante debía autoevaluar su nivel de implicación y percepción de aprendizaje usando una rúbrica previamente consensuada. Como producto final del proyecto, los estudiantes del Centro B diseñaron posters para presentar sus investigaciones de manera oral en unas jornadas científicas en el instituto. Además, cada estudiante debía realizar un vídeo en el que se mostraba y explicaba su experimento. Estos productos fueron evaluados por los docentes y coevaluados por los propios estudiantes mediante rúbricas.

\section{Dos proyectos de naturaleza diferente}

La concreción de los elementos de diseño de cada proyecto en ambos centros resultó en dos actividades de naturaleza y enfoque diferente, particularmente respecto al grado de autenticidad de la indagación. Mientras que el proyecto del congreso científico del Centro A se llevó a cabo una investigación de carácter teórico que hizo énfasis en las características formales y la parte comunicativa final, el proyecto en el Centro B hizo énfasis en la promoción de una indagación empírica, abierta y libre. En la Tabla 1 se describen las características principales de cada versión tomando como referencia los elementos esenciales de la metodología ABP aplicada a las ciencias descritos por Hasni et al. (2016), con la finalidad de poder ofrecer una mejor interpretación de los datos obtenidos. 
Tabla 1. Resumen de los elementos de diseño del proyecto "el congreso científico" de los dos centros participantes, según las características de proyectos ABP de ciencias de Hasni et al. (2016).

\begin{tabular}{|c|c|c|}
\hline $\begin{array}{l}\text { Elementos de } \\
\text { diseño del proyecto }\end{array}$ & Centro A & Centro B \\
\hline $\begin{array}{l}\text { Autenticidad del } \\
\text { problema o la } \\
\text { pregunta científica }\end{array}$ & $\begin{array}{l}\text { El alumnado selecciona y refina una } \\
\text { pregunta a partir de un conjunto } \\
\text { propuesto por el profesorado, pudiendo } \\
\text { dar lugar a trabajos diferentes según el } \\
\text { enfoque que asuma el grupo. }\end{array}$ & $\begin{array}{l}\text { El alumnado propone y refina una } \\
\text { pregunta investigable de acuerdo con sus } \\
\text { propios intereses (autenticidad personal). }\end{array}$ \\
\hline $\begin{array}{l}\text { Implicación } \\
\text { alumnado en la } \\
\text { investigación } \\
\text { científica o el diseño } \\
\text { de actividades }\end{array}$ & $\begin{array}{l}\text { Cada grupo de trabajo (4-5 estudiantes) } \\
\text { concreta y refina el enfoque de la } \\
\text { pregunta inicial. Los grupos realizan una } \\
\text { búsqueda bibliográfica iterativa hasta } \\
\text { encontrar una solución satisfactoria a la } \\
\text { pregunta. Se usan estándares de calidad } \\
\text { reales (revisión crítica de la bibliografía, } \\
\text { estilo de citación...). }\end{array}$ & $\begin{array}{l}\text { En parejas, los estudiantes deben } \\
\text { responder a la pregunta inicial mediante el } \\
\text { diseño de un experimento, la obtención y } \\
\text { el análisis de datos, su representación y la } \\
\text { elaboración de explicaciones científicas. } \\
\text { Cada pareja de trabajo diseña todo el } \\
\text { proceso de indagación (indagación } \\
\text { auténtica). }\end{array}$ \\
\hline $\begin{array}{l}\text { Elaboración de un } \\
\text { producto o artefacto } \\
\text { final }\end{array}$ & $\begin{array}{l}\text { Defensa de la respuesta frente a tribunal } \\
\text { formado por docentes del centro y } \\
\text { personas del ámbito universitario expertas } \\
\text { en la materia investigada. }\end{array}$ & $\begin{array}{l}\text { Se realiza un póster y un video-resumen de } \\
\text { la investigación que se expone al resto de } \\
\text { docentes y alumnado del centro. }\end{array}$ \\
\hline Colaboración & $\begin{array}{l}\text { Además del trabajo en grupo, se realiza } \\
\text { un seguimiento informal durante el } \\
\text { diálogo de clase, así como se establecen } \\
\text { sesiones semanales de 'parada' con el fin } \\
\text { de evaluar el estado de las investigaciones } \\
\text { y estructurar la reflexión del alumnado. Se } \\
\text { establecen entregas parciales del proyecto } \\
\text { con retorno de evaluación formativa por } \\
\text { parte del profesorado. }\end{array}$ & $\begin{array}{l}\text { La reflexión se produce de manera } \\
\text { informal durante el diálogo de clase y } \\
\text { mediante el uso de rúbricas de } \\
\text { autoevaluación en los últimos minutos de } \\
\text { cada clase. Se usan rúbricas de } \\
\text { coevaluación del producto final. }\end{array}$ \\
\hline $\begin{array}{l}\text { Uso de tecnologías } \\
\text { digitales para el } \\
\text { aprendizaje }\end{array}$ & $\begin{array}{l}\text { Se usan tecnologías digitales para acceder } \\
\text { a la información, así como para encontrar, } \\
\text { elaborar y comunicar el producto final. }\end{array}$ & $\begin{array}{l}\text { Se usan tecnologías digitales para elaborar } \\
\text { los datos empíricos obtenidos y elaborar y } \\
\text { comunicar el producto final. }\end{array}$ \\
\hline
\end{tabular}

\section{Instrumentos y procedimiento para la toma de datos}

Este estudio se basa en una metodología cuantitativa descriptiva, con un diseño ex post facto. Se optó por este enfoque puesto que la finalidad fue obtener datos sobre el máximo número de estudiantes para poder establecer relaciones entre centro y género. Así, para dar respuesta a la pregunta de investigación, se diseñó un cuestionario para el alumnado de ambos centros participante del proyecto "El congreso científico". En particular, el cuestionario pretendía obtener información sobre la autoeficacia percibida por el alumnado en relación con cada una de las 7 prácticas científicas definidas por Osborne (2014). La redacción de los ítems se basó en herramientas similares usadas y validadas, como las publicadas en Grimalt-Álvaro y Couso (2018), siguiendo las indicaciones A. Bandura (2012) y Bong y Skaalvik (2003), es decir, en términos de capacidad (p. ej. Puedo hacerlo) en lugar de expectativas de futuro (p. ej. Podré hacerlo) y en relación con el dominio de cada práctica en cuestión y para una actividad concreta. El cuestionario fue revisado y refinado iterativamente en diversos ciclos donde participaron investigadores/as del equipo y posteriormente investigadores/as externos, que evaluaron la validez del contenido (por ejemplo, evaluando la expresión clara y precisa de las preguntas y respuestas, la relevancia de las variables consideradas en relación con la literatura, o un tiempo de respuesta razonable, entre otros). 
El redactado básico final de la pregunta se muestra a continuación:

¿Hasta qué punto sientes que eres capaz de llevar a cabo de manera satisfactoria cada una de las siguientes prácticas?

Diseñar experiencias para tomar datos (P1)

Formular hipótesis o predicciones sobre un experimento (P2)

Identificar qué factores o condiciones (variables) pueden afectar a un experimento (P3)

Tomar datos y representarlos gráficamente (P4)

Dar una explicación cientifica a fenómenos o experiencias (P5)

Obtener conclusiones a partir de unos datos tomados (P6)

Argumentar en público los resultados de una investigación (P7)

Para facilitar la respuesta al alumnado, cada ítem relacionado con cada práctica científica se acompañó de diversos ejemplos concretos relacionados con el proyecto, aunque por motivos de espacio no se muestran en el presente artículo. Las respuestas a los ítems se recogieron mediante el uso de una escala Likert de 6 puntos (no me siento capaz - me siento totalmente capaz y podría ayudar a mis compañeros y compañeras), en línea con los estudios de Kier et al. (2014) y G. Thomas et al. (2008). La fiabilidad final se evaluó con el coeficiente alfa de Cronbach, mostrando buenos niveles de consistencia interna a nivel general $(0,89)$ y para las 7 subescalas consideradas $(\mathrm{P} 1=0,88 ; \mathrm{P} 2=0,86 ; \mathrm{P} 3=0,86 ; \mathrm{P} 4=0,87 ; \mathrm{P} 5=0,86 ; \mathrm{P} 6=0,87 ; \mathrm{P} 7=0,89)$. El cuestionario se distribuyó inmediatamente antes y después de la realización del proyecto del congreso científico.

De manera complementaria, se incluyeron diversas preguntas para conocer las características personales de cada estudiante, como el género, centro de pertenencia, o posible repetición de curso, de respuesta cerrada. En el cuestionario posterior, se añadió una sección final destinada a conocer la percepción del alumnado sobre qué elemento/s había/n tenido mayor impacto a la hora de hacerles sentir más capaces de hacer ciencia, de respuesta abierta, con preguntas como las que se reproducen a continuación:

Durante el congreso, ¿qué es lo que te ha hecho sentir que eras capaz de llevar a cabo una investigación cientifica? (puedes explicarnos algún ejemplo o situación que creas que te baya becho sentir asi) (P10)

¿Cuál de tus potencialidades (lo que crees que se te da bien, en lo que eres bueno/a) crees que te ba sido útil para llevar a cabo la investigación científica del congreso? (P11)

De los 109 estudiantes que respondieron los cuestionarios pre y post, para el estudio sólo se han tenido en cuenta los estudiantes que pudieron ser identificados en ambos cuestionarios, formando una muestra final de 60 estudiantes (27 chicas y 33 chicos), para los dos centros: 26 respuestas para el Centro A (10 chicos y 16 chicas) y 34 respuestas para el Centro B (23 chicos y 11 chicas).

\section{Análisis de datos}

El análisis de los datos obtenidos en la encuesta se realizó en dos etapas usando el programa JASP versión 0.12.2. En la primera etapa se realizó un análisis descriptivo para obtener una perspectiva general de los datos, a partir del recuento y establecimiento de porcentajes. En esta misma etapa se realizaron pruebas de normalidad para evaluar la distribución de las respuestas en cada variable tipo Likert. En una segunda etapa, se realizó un análisis bivariado para identificar diferencias en las respuestas para cada centro y género. Puesto que los datos no se ajustaron bien a una distribución normal, se optó por la selección de pruebas no paramétricas para dar respuesta a la pregunta de investigación. Así, para identificar posibles desigualdades iniciales en la percepción de autoeficacia entre chicos y chicas de cada centro (muestras 
independientes) se usó la prueba $U$ de Mann-Whitney con un nivel de confianza del 95\%. Para analizar las diferencias entre la autoeficacia pre y post de chicos y chicas para cada centro (muestras apareadas) se usó la prueba de los rangos con signo de Wilcoxon de una cola con un nivel de confianza del 95\%, siguiendo a Somekh y Lewin (2005). De manera adicional, se calculó la correlación biserial por rangos para todos los contrastes realizados, como una evaluación no paramétrica de la medida del efecto observado, es decir, de la magnitud de la posible diferencia y la fuerza de la relación entre las variables contrastadas, en línea con Tomczak y Tomczak (2014). Cuanto más cercana a 1 sea el resultado de la correlación, mayor será el efecto.

Las respuestas abiertas finales del alumnado sobre los elementos que ayudaron a mejorar su autoeficacia se usaron para contrastar y aumentar la validez de la interpretación de los resultados obtenidos en las pruebas estadísticas descritas. Mediante un análisis del contenido, las respuestas fueron categorizadas según su relación con los cuatro ámbitos de influencia definidos por Bandura (1997).

\section{Resultados y discusión}

\section{Análisis del nivel inicial de autoeficacia en ciencias del alumnado participante}

En la Figura 2 se muestran los niveles de autoeficacia inicial de los estudiantes de ambos centros para cada una de las 7 prácticas científicas consideradas. Tal y como se observa, los niveles de autoeficacia iniciales raramente superan el nivel "me siento bastante capaz" para el Centro A y se encuentran más cerca del nivel "me siento moderadamente capaz" en el Centro B. En ambos centros los valores más altos de autoeficacia se encuentran en la práctica P4 y P6. En cambio, los niveles iniciales más bajos se encuentran en la P7 y P3.

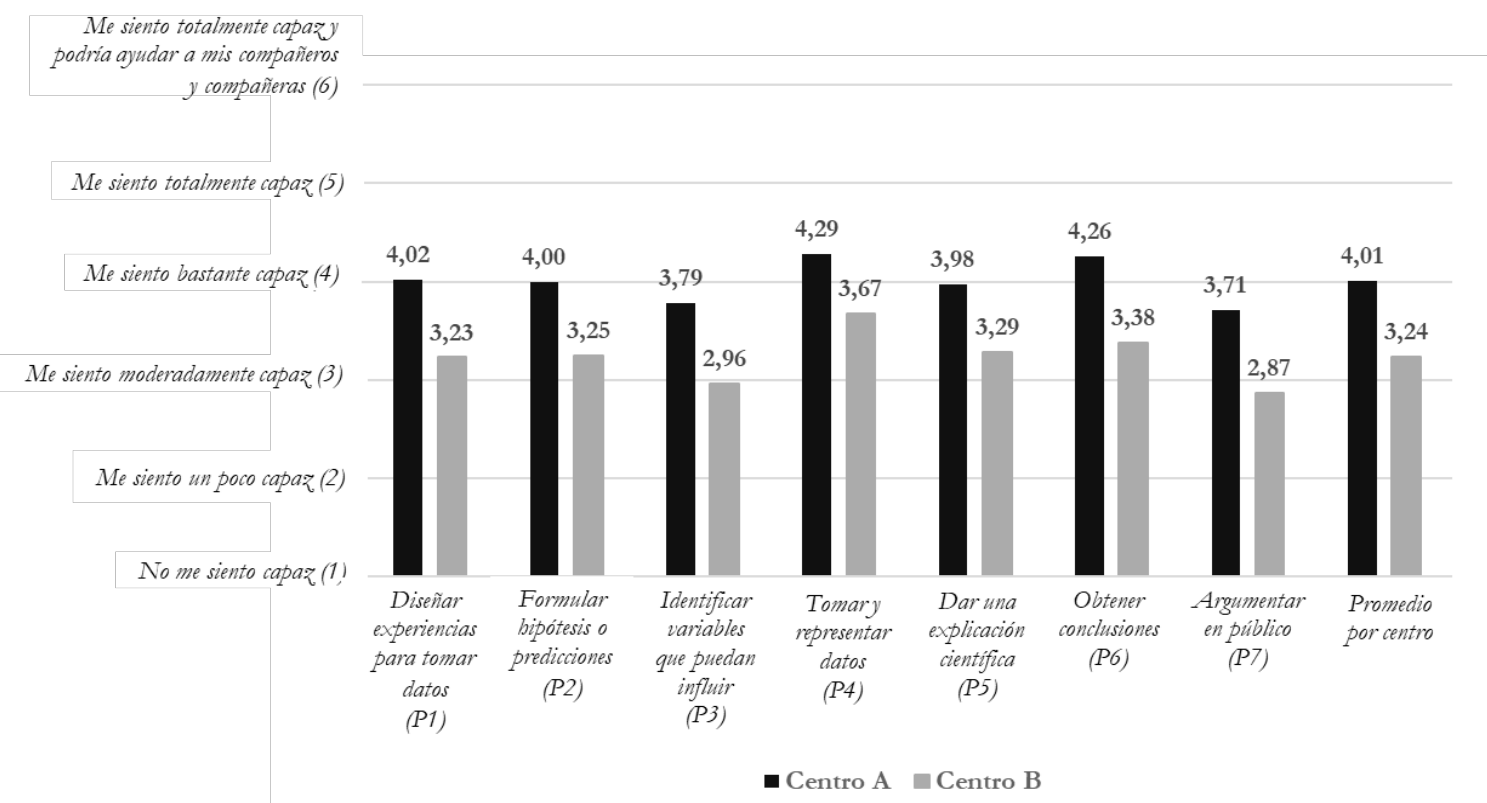

Figura 2. Promedio del nivel de autoeficacia inicial del alumnado del Centro A $(N=26)$ y Centro $B(N=34)$ para cada práctica científica.

Aunque para ambos centros el alumnado siente que tiene más o menos capacidades en las mismas prácticas, el alumnado del Centro A puntuó sistemáticamente por encima del alumnado del Centro B para todas las prácticas. Según Webb-Williams (2018), estas diferencias podrían explicarse por la diferente tipología de centro (privado y público) así como por la alta proporción de alumnado con necesidades específicas de apoyo educativo en el Centro B, que podría influir de manera indirecta en la autoeficacia del alumnado de este Centro B. Aunque sería necesario ahondar más en un estudio comparativo para extraer conclusiones pertinentes, que no es el 
objeto de la presente investigación, esta desigualdad inicial en la autoeficacia del alumnado de ambos centros, juntamente con las diferentes características de los elementos de diseño del proyecto científico, anuncia una evolución distinta de la autoeficacia del alumnado participante.

Para analizar las diferencias en la autoeficacia inicial del alumnado, se realizaron contrastes entre los niveles de autoeficacia para cada práctica científica, género y centro mediante la prueba $U$ de Mann-Whitney de dos colas. Los resultados de estos contrastes (Anexo 1) no revelan diferencias iniciales estadísticamente significativas entre cómo los chicos y las chicas de cada centro percibían sus propias habilidades para cada práctica científica y para $p<0,050$. Los resultados de las correlaciones de rangos muestran que las relaciones entre variables son débiles o moderadas y, por lo tanto, sustentan la ausencia de diferencias estadísticamente significativas. Así, tanto los chicos como las chicas de ambos centros se puntuaron con un nivel de autoeficacia relativamente bajo de manera similar. Estos resultados contrastan con los resultados de la literatura, donde se ha reportado ampliamente que las chicas suelen infravalorarse en el ámbito científico en comparación con sus compañeros, que suelen sobrevalorarse (Huang, 2013; Webb-Williams, 2018). Cabe destacar que estas diferencias podrían haber quedado sin evidenciar debido al reducido tamaño de los grupos resultantes segregados por centro y género cuando se realiza el contraste. De este modo, cabría esperar que emergieran más diferencias si se repitiera el estudio con una muestra mayor. El esfuerzo de ambos centros por promover estrategias de autorregulación y aprendizaje autónomo no sólo en el ABP, sino a lo largo de toda la educación secundaria también podría explicar por qué es difícil encontrar diferencias en el nivel de autoeficacia en estos estudiantes, puesto que chicos y chicas serían más realistas a la hora de valorarse, minimizando las diferencias según el género.

\section{Variación de la autoeficacia en el alumnado del Centro A}

Los resultados de la variación de la autoeficacia a corto plazo del alumnado del Centro A antes y después de participar en el proyecto del congreso científico se realizaron de manera segregada por género y se muestran en la Tabla 2. A nivel general, se observa un aumento en el valor de la autoeficacia del alumnado para múltiples prácticas científicas, tanto a nivel de significancia como a nivel de la correlación biserial por rangos, puesto que las diferencias entre los niveles reportados de autoeficacia son significativamente superiores en la mayoría de los casos $(p<0,05 \mathrm{y}$ valores mayores de 0,5 en la correlación).

Tabla 2. Resultados de los contrastes de muestras apareadas mediante la prueba de los rangos con signo de Wilcoxon para el Centro A (Nchicos $=10 \mathrm{y}$ Nchicas=16). Para todas las pruebas, la hipótesis alternativa especifica que la medida 1 (pre) es menor que la medida 2 (post). Las filas que se encu entran sombreadas señalan aquellos casos en los que la diferencia entre la autoeficacia post- y la pre- es estadísticamente significativa.

\begin{tabular}{|c|c|c|c|c|}
\hline \multicolumn{2}{|l|}{ Diferencias PRE-POST Centro A } & \multirow{2}{*}{$\begin{array}{c}\begin{array}{c}\text { Valor del } \\
\text { estadístico } \\
\text { (W) }\end{array} \\
8,000\end{array}$} & \multirow{2}{*}{$\begin{array}{c}\begin{array}{c}\text { Valor de la } \\
\text { significancia } \\
(\boldsymbol{p})\end{array} \\
0,333 \\
\end{array}$} & \multirow{2}{*}{$\begin{array}{c}\begin{array}{c}\text { Correlación } \\
\text { biserial por } \\
\text { rangos }\end{array} \\
-0,238 \\
\end{array}$} \\
\hline \multirow{2}{*}{ P1: Diseñar experiencias para tomar datos } & Chicos & & & \\
\hline & Chicas & 11,000 & 0,026 & $-0,667$ \\
\hline \multirow{2}{*}{$\begin{array}{l}\text { P2: Formular hipótesis o predicciones } \\
\text { sobre un experimento }\end{array}$} & Chicos & 3,500 & 0,035 & $-0,750$ \\
\hline & Chicas & 5,000 & 0,037 & $-0,722$ \\
\hline \multirow{2}{*}{$\begin{array}{l}\text { P3: Identificar qué factores o condiciones } \\
\text { (variables) pueden afectar a un experimento }\end{array}$} & Chicos & 16,500 & 0,114 & $-0,400$ \\
\hline & Chicas & 3,000 & 0,010 & $-0,867$ \\
\hline \multirow{2}{*}{$\begin{array}{l}\text { P4: Tomar datos y representarlos } \\
\text { gráficamente }\end{array}$} & Chicos & 9,000 & 0,111 & $-0,500$ \\
\hline & Chicas & 7,000 & 0,062 & $-0,611$ \\
\hline \multirow{2}{*}{$\begin{array}{l}\text { P5: Dar una explicación científica a } \\
\text { fenómenos o experiencias }\end{array}$} & Chicos & 16,000 & 0,414 & $-0,111$ \\
\hline & Chicas & 45,500 & 0,335 & $-0,133$ \\
\hline \multirow{2}{*}{$\begin{array}{l}\text { P6: Obtener conclusiones a partir de unos } \\
\text { datos tomados }\end{array}$} & Chicos & 2,000 & 0,043 & $-0,810$ \\
\hline & Chicas & 18,000 & 0,304 & $-0,200$ \\
\hline \multirow{2}{*}{$\begin{array}{l}\text { P7: Argumentar en público los resultados } \\
\text { de una investigación }\end{array}$} & Chicos & 0,000 & 0,007 & $-1,000$ \\
\hline & Chicas & 10,000 & 0,040 & $-0,64$ \\
\hline
\end{tabular}


En el Centro A, después de participar en el congreso científico tanto los chicos como las chicas se sintieron más capaces de llevar a cabo predicciones o hipótesis (P2, Tabla 2). Este resultado contrasta con el carácter de la indagación planteada en el proyecto: si bien en el Centro A no se realizó una indagación empírica (relacionada con la experiencia con un fenómeno real), tanto chicos como chicas se sienten más capaces de formular hipótesis o predicciones sobre un experimento. No obstante, el hecho de que el alumnado haya tenido acceso a una cantidad grande de información de diversos experimentos reales hechos previamente, en los que se reportan las respectivas predicciones o hipótesis de manera simplificada, puede haber contribuido a aumentar la percepción de capacidad del alumnado para realizar la misma tarea en un escenario futuro. Esta misma situación podría relacionarse con el aumento de la autoeficacia en la práctica $\mathrm{P} 6$ para los chicos, y las prácticas $\mathrm{P} 1$ y P3 para las chicas (Tabla 2). Así pues, tener información puede devenir una estrategia positiva a la hora de aumentar la autoeficacia del alumnado en ciencias, puesto que promueve que éste conozca mejor el fenómeno y la interpretación científica del mismo y se familiarice con el fenómeno de estudio en línea con Jiménez-Liso (2020), siendo un paso previo necesario a la indagación empírica. Esta característica del congreso científico del Centro A también es reconocida por el alumnado como aquello que mayoritariamente les había hecho sentir que eran capaces de llevar a cabo una investigación científica en las respuestas abiertas al cuestionario (buscar información sobre el tema que nos ha tocado hacer, saber buscar información; encontrar información y aportar ideas...).

Por otro lado, se observa un aumento significativo de la autoeficacia en la práctica P7 tanto para chicos como para chicas (Tabla 2). Este aumento, totalmente relacionado con la orientación hacia la exposición pública del proyecto en el Centro A, es mayor en los chicos que en las chicas, puesto que los resultados en la significancia $(p)$ y la correlación biserial son mucho mejores. Estos resultados muestran otra de las diferencias de género que se pueden identificar en el aula: mientras que la competencia lingüística es significativamente mayor en las chicas que en los chicos según los resultados de las pruebas PISA (OECD, 2019), los chicos están más habituados a dominar e intervenir en el espacio público y las chicas suelen tener una menor presencia pública (Archer et al., 2016). Así, la alineación entre la práctica científica y la práctica social atribuida al género masculino explicaría la mayor variación de la autoeficacia en los chicos, siendo independiente de la competencia real. Ciertamente, a la hora de promover una actividad inclusiva, implementar estrategias como la obligación de que todos los miembros del grupo hablen, como sucedió en el proyecto del Centro A, permite equilibrar la presencia pública de chicos y chicas. No obstante, cabe tener en cuenta que, para las chicas, esta estrategia puede forzar una situación incómoda -más incómoda que la que pueda representar para sus compañeros- tal y como se refleja en el aumento diferente de la autoeficacia. Por lo tanto, aunque el equilibrio de la participación pueda parecer efectivo, para acompañar a las chicas a tener una mayor presencia en el espacio público es necesario tener en cuenta y conseguir modelar la influencia de factores que afectan especialmente la autoeficacia de las chicas, como es el alto nivel de importancia de la opinión de los demás (docentes y compañeros/as) sobre el propio desempeño (Bryant, 2017).

Cabe añadir que no se observan diferencias estadísticamente significativas para la práctica P4 para los chicos y chicas del Centro A. Desde nuestra perspectiva, la complejidad de los diseños de los experimentos consultados (instrumentación utilizada, interpretación de datos complejos...) no contribuiría a que el alumnado sintiera que puede llevar a cabo una tarea similar. Cabe tener en cuenta que, aunque la autoeficacia tiene una orientación hacia un posible resultado futuro, se basa en experiencias previas que sirven de referencia (Bandura, 1997). Por este motivo, deviene esencial promover experiencias de éxito en tareas similares o proveer de ejemplos cercanos y parecidos que contribuyan a crear una base de referencia 
sólida. Un ejemplo lejano (como lo puede ser el diseño de un experimento científico real) dificulta que pueda servir como referencia de éxito para que el alumnado sienta que puede llevar a cabo una tarea similar. Estos resultados no sólo muestran la necesidad de proveer buenos referentes, sino también la necesidad de ofrecer un buen andamiaje en la indagación basado en la resolución de pequeños retos asumibles para el alumnado.

\section{Variación de la autoeficacia del alumnado del Centro B}

En la Tabla 3 se muestran los contrastes entre el nivel inicial y final de autoeficacia reportado por el alumnado del Centro B, segregado por género. A diferencia del Centro A, el impacto del proyecto del congreso científico a corto plazo en el alumnado del Centro B sólo se observa para los chicos y en relación con la práctica P2 (formular hipótesis o predicciones sobre un experimento).

Tabla 3. Resultados de los contrastes de muestras apareadas mediante la prueba de los rangos con signo de Wilcoxon para el Centro B, donde Nchicos=23 y Nchicas=11. Para todas las pruebas, la hipótesis alternativa especifica que la medida 1 (pre) es menor que la medida 2 (post). Las filas que se encuentran sombreadas señalan aquellos casos en los que la diferencia entre la autoeficacia post- y la pre- es estadísticamente significativa.

\begin{tabular}{|c|c|c|c|c|}
\hline \multicolumn{2}{|l|}{ Diferencias PRE-POST Centro B } & \multirow{2}{*}{$\begin{array}{c}\begin{array}{c}\text { Valor del } \\
\text { estadístico } \\
\text { (W) }\end{array} \\
33,000\end{array}$} & \multirow{2}{*}{$\begin{array}{c}\begin{array}{c}\text { Valor de la } \\
\text { significancia } \\
(p)\end{array} \\
0,194\end{array}$} & \multirow{2}{*}{$\begin{array}{c}\begin{array}{c}\text { Correlación } \\
\text { biserial por } \\
\text { rangos }\end{array} \\
-0,275\end{array}$} \\
\hline \multirow{2}{*}{ P1: Diseñar experiencias para tomar datos } & Chicos & & & \\
\hline & Chicas & 5,000 & 0,140 & $-0,524$ \\
\hline \multirow{2}{*}{$\begin{array}{l}\text { P2: Formular hipótesis o predicciones } \\
\text { sobre un experimento }\end{array}$} & Chicos & 34,000 & 0,004 & $-0,642$ \\
\hline & Chicas & 9,000 & 0,215 & $-0,357$ \\
\hline \multirow{2}{*}{$\begin{array}{l}\text { P3: Identificar qué factores o condiciones } \\
\text { (variables) pueden afectar a un experimento }\end{array}$} & Chicos & 40,500 & 0,126 & $-0,325$ \\
\hline & Chicas & 10,500 & 0,294 & $-0,250$ \\
\hline \multirow{2}{*}{ 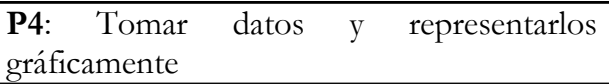 } & Chicos & 40,500 & 0,071 & $-0,404$ \\
\hline & Chicas & 12,000 & 0,112 & $-0,467$ \\
\hline \multirow{2}{*}{$\begin{array}{l}\text { P5: Dar una explicación científica a } \\
\text { fenómenos o experiencias }\end{array}$} & Chicos & 25,500 & 0,254 & $-0,227$ \\
\hline & Chicas & 30,000 & 0,625 & 0,091 \\
\hline \multirow{2}{*}{$\begin{array}{l}\text { P6: Obtener conclusiones a partir de unos } \\
\text { datos tomados }\end{array}$} & Chicos & 30,000 & 0,401 & $-0,091$ \\
\hline & Chicas & 2,500 & 0,102 & $-0,667$ \\
\hline \multirow{2}{*}{$\begin{array}{l}\text { P7: Argumentar en público los resultados } \\
\text { de una investigación }\end{array}$} & Chicos & 48,500 & 0,090 & $-0,366$ \\
\hline & Chicas & 22,500 & 0,526 & 0,000 \\
\hline
\end{tabular}

En este caso, la indagación propuesta fue completamente abierta, puesto que el alumnado debía definir una pregunta de investigación y desarrollar un proceso completo de indagación empírica para responderla. En la mayoría de los casos, esta apertura dio la oportunidad al alumnado de realizar preguntas originales y relacionadas con su vida cotidiana, pero poco investigables que tuvieron que ser refinadas progresivamente con la ayuda de las docentes. Esta situación, juntamente con el hecho de no disponer de un nivel de familiaridad adecuado con el fenómeno de estudio (Jiménez-Liso, 2020), ni contar con referencias previas de indagaciones similares, explicaría por qué la mejora de la autoeficacia del alumnado después de la participación en el proyecto es escasa, en línea con Ferrés et al. (2015). Las expectativas iniciales del alumnado no pudieron cumplirse en este caso y, por lo tanto, podría decirse que el alumnado del Centro B fue demasiado optimista al inicio, como se describe en una situación equivalente en Atadero et al. (2015). La facilidad de los chicos para evaluar de manera positiva su nivel de desempeño, reportada en Huang, (2013) y Webb-Williams (2018), explicaría por qué la única situación en la que se identifica un aumento de la autoeficacia estadísticamente significativo es en un grupo de chicos (P2, Tabla 3).

En comparación con el Centro A, el grado de apertura y autenticidad de la indagación fue mayor en el proyecto del Centro B, lo que supuso también un reto superior para el alumnado de este centro. Para superar este reto, la figura del docente como referencia del contenido y a 
la hora de proporcionar la ayuda necesaria para avanzar en la investigación ganó relevancia, según se refleja en las opiniones del alumnado: Yo mismo me propuse superar el reto con la ayuda de mi profesor; la ayuda de mis compañeros/as y de los profesores [me hizo sentir capar]; la ayuda de los profesores a la hora de realizar hipótesis y sacar conclusiones[me bizo sentir capaz]; El trabajo en grupo también es un potencial; pienso que me faltó mucha ayuda de mis profesores. Esta situación explica también la ausencia observada en el aumento de la autoeficacia del alumnado, puesto que los y las estudiantes habrían sentido que sin la aportación del docente (y de sus compañeros/as) no habrían sido capaces de avanzar. Por lo tanto, en esta situación el éxito conseguido es atribuido a la participación de los demás, y no a las propias capacidades, tal y como también argumenta Bandura (1997), lo que anula el efecto de fuente de autoeficacia de la experiencia educativa.

\section{Conclusiones e implicaciones}

En este trabajo se ha analizado el efecto de dos planteamientos con diferentes grados de autenticidad en la indagación científica en la mejora de la autoeficacia en las ciencias a corto plazo de los y las estudiantes de secundaria participantes en dos proyectos de ciencias. En el Centro A, el alumnado debía responder a una pregunta guía mediante una búsqueda y análisis bibliográfico de diversas fuentes y exponer las conclusiones delante de un tribunal externo. La implementación conllevó un aumento del nivel de autoeficacia tanto para los chicos como las chicas en diversas prácticas científicas, que se relaciona con el hecho de que, a nivel de la indagación planteada, el reto fuera familiar y no novedoso para el alumnado (había realizado otras búsquedas bibliográficas anteriormente) o que el reto fuera cercano y más alcanzable de manera autónoma, puesto que el alumnado contaba con referencias externas.

En este mismo centro, se observó una mejora muy superior de la autoeficacia de los chicos en la argumentación en público (P7), en comparación con las chicas. Esta diferencia se relaciona con una alineación de esta práctica científica con la práctica social atribuida al rol masculino (dominio del espacio público) y la necesidad de implementar estrategias apropiadas para balancear una participación equitativa y respetuosa de chicos y chicas. Con esta finalidad, deviene necesario ir más allá de promover una distribución de tiempo igualitaria, para implementar estrategias que permitan crear un ambiente de aprendizaje seguro. Una de las más relevantes es el análisis y cuestionamiento de las propias prácticas docentes para evitar los estereotipos de género en el aula (por ejemplo, usando lenguaje no sexista para dirigirse al grupo de estudiantes en general y/o a chicos o chicas, usando lenguaje no sexista para describir profesiones y roles en el ámbito científico, ser consciente de los estereotipos de género que existen sobre las disciplinas científicas para compensarlos activamente...) así como promover una interacción respetuosa entre el alumnado (también a través del uso no sexista del lenguaje, fomentando la reflexión personal antes de intervenir en el aula, o distribuyendo roles entre el alumnado de manera equitativa) (Dewsbury y Brame, 2019; Tanner, 2013; The GiST project 2020).

En el Centro B, el alumnado debía plantear una pregunta investigable, diseñar una indagación para tomar datos y responderla, y presentar la investigación delante de sus compañeros/as y docentes. Aunque el nivel de autenticidad de la indagación fuera mayor, prácticamente no se observó variación en la autoeficacia del alumnado. Esta situación se relaciona con la novedad de la propuesta (el alumnado contaba con pocos referentes personales o externos previos y debía "construir de cero"), una mayor complejidad del reto debido al alto grado de apertura de la indagación (puesto que el alumnado tuvo responsabilidad y poder de decisión en todas las etapas de la investigación) y de las limitaciones del alcance de la guía proporcionada por los docentes, al ser estos la única referencia externa y haber muchas temática y problemáticas distintas (diversidad). Esta combinación de características dificultaría que el alumnado 
atribuyera el éxito a sus propias capacidades, disminuyendo el potencial impacto positivo en la autoeficacia de la propuesta educativa.

Aunque la implicación del alumnado en indagaciones auténticas pueda tener un interés a la hora de promover actitudes positivas y competencias en el ámbito, la implementación de las dos versiones del proyecto muestra que un mayor grado en la autenticidad de la práctica científica no deriva necesariamente en una mayor autoeficacia en ciencias en el alumnado participante, en línea con Ferrés et al. (2015). La experiencia descrita en este artículo muestra cómo en el diseño de proyectos educativos habrá que resolver dos tensiones en la búsqueda de estrategias que promuevan la autoeficacia en el alumnado. La primera, referente al grado de familiaridad - novedad del alumnado con la propuesta planteada tanto a nivel conceptual, como en relación con todas las etapas del ciclo de indagación (Jiménez-Liso, 2020): más familiaridad posibilita disponer de más referentes personales y una mayor repercusión en la autoeficacia, pero menor interés y curiosidad.

La segunda tensión se encuentra en el grado de complejidad o alta demanda - simplicidad de la propuesta planteada. Un nivel alto de complejidad posibilita la apertura de diversas etapas de indagación y la incorporación de datos empíricos a la indagación; pero también implica el diseño de un andamiaje mucho más elaborado que haga uso de diversos recursos (verbales, físicos o digitales) en cada etapa de la indagación para que cada estudiante disponga de los elementos necesarios para culminar con éxito su proyecto. Con esta finalidad, existen diversas estrategias que pueden ir desde la proporción de pistas, instrucciones o explicaciones, a la formulación de preguntas, modelización o retroalimentación, como describen Crujeiras Pérez y Jiménez Aleixandre (2018). Como la formulación de preguntas suele ser la estrategia más frecuente en el aula, Mergendoller et al. (2006) recomiendan el uso de preguntas abiertas para facilitar el pensamiento crítico y la autonomía del alumnado (resume dónde te encuentras abora mismo ¿Cómo sabes eso? ¿Dónde estás atrapado/ a? ¿Qué crees que sería útil saber abora? ¿Dónde puedes encontrar la información que necesitas?). A medida que el alumnado adquiere experiencia y capacidad para llevar a cabo la tarea, el docente va cediendo progresivamente el control sobre el proceso de aprendizaje. Así, deviene necesario disponer de herramientas adecuadas para evaluar el nivel de competencia de indagación del alumnado para seleccionar la cantidad de andamiaje necesaria (por ejemplo, a partir de la herramienta NPTAI propuesta por Ferrés et al. (2015). Del mismo modo, para que el alumnado desarrolle progresivamente las competencias necesarias para realizar una indagación cada vez más compleja, es necesario promover la reflexión sobre el propio proceso de aprendizaje. El uso de rúbricas, como se usaron en ambos centros, puede ser un instrumento con grandes potencialidades, puesto que proporciona información que ayuda a los estudiantes a comprender lo que saben y lo que no saben, así como orientación sobre lo que necesitan aprender (Capraro y Corlu, 2013). Estos autores también proponen que, para fomentar el desarrollo de las competencias metacognitivas, es importante que el alumnado participe en el desarrollo de las rúbricas, sea reflexivos aprendiendo a autoevaluarse, y sepan recibir comentarios críticos y constructivos de compañeros/as (coevaluación). En definitiva, proporcionar un andamiaje adecuado y promover estrategias de autorregulación es imprescindible para un desarrollo correcto de la autoeficacia en el alumnado (Lai et al., 2018).

Cabe añadir que mostrar un nivel adecuado de autoeficacia no significa que el alumnado sepa realizar dicha tarea $\mathrm{y}$, por consiguiente, sigue siendo necesario garantizar buenas oportunidades de aprendizaje. El abuso de retos excesivamente sencillos y familiares no resulta en una buena estrategia educativa puesto que, aunque la autoeficacia pueda aumentar, se pierden oportunidades para promover el desarrollo de la competencia real del alumnado en la práctica científica. Desarrollar un nivel adecuado de autoeficacia, debidamente regulado por experiencias de referencia, es necesario como precursor de la acción. Es decir, para que el 
alumnado se involucre en la práctica científica, debe sentir que tiene las capacidades para hacerlo. Para determinados colectivos en desventaja en el ámbito científico, como estudiantes de nivel socioeconómico bajo, minorías étnicas, o chicas, esta situación toma una especial relevancia, puesto que suelen tener unos niveles de autoeficacia significativamente más bajos que sus compañeros (Archer y DeWitt, 2015). Estrategias como proveer buenos referentes, familiarizar el alumnado con el fenómeno, y balancear de manera respetuosa la participación en el espacio público pueden contribuir a ofrecer un buen andamiaje o apoyo en la indagación $\mathrm{y}$, por consiguiente, ayudar a dimensionar los retos planteados al alumnado para que sean asumibles y puedan convertirse no sólo en experiencias de éxito para todos y todas, sino en oportunidades de aprendizaje que empoderan.

\section{Limitaciones}

Una de las limitaciones principales del presente estudio es en relación con el tamaño pequeño de la muestra usado, puesto que puede haber ocultado alguna relación entre las variables de estudio. La réplica de este estudio en una muestra mayor de alumnado contribuirá al refinamiento de los resultados, así como a la identificación de otras posibles variables relevantes que hayan podido influir en los resultados y que no se han considerado en el presente estudio. En futuras investigaciones puede ser conveniente incorporar metodologías cualitativas (entrevistas, diarios de aprendizaje...) que ayuden a explorar y complementar la relación entre el grado de autenticidad de la indagación en los proyectos de ciencias y la autoeficacia del alumnado.

\section{Agradecimientos}

Para la realización de la investigación se ha obtenido apoyo del Ministerio de Economía y Competitividad (PGC2018-096581-B-C21) así como del grupo ACELEC (2017SGR1399) y del grupo ARGET (2017SGR1682).

\section{Referencias}

Archer, L., Dawson, E., Seakins, A., DeWitt, J., Godec, S., y Whitby, C. (2016). "I'm Being a Man Here": Urban Boy's Performances of Masculinity and Engagement With Science During a Science Museum Visit. Journal of the Learning Sciences, 25(3), 438-485.

Archer, L., Osborne, J., DeWitt, J., Dillon, J., Wong, B., y Willis, B. (2013). ASPIRES. Young people's science and career aspirations, age 10-14.

Archer, L., y Dewitt, J. (2015). Science Aspirations and Gender Identity: Lessons from the ASPIRES Project. In E. K. Henriksen, J. Dillon, y J. Ryder (Eds.), Understanding Student Participation and Choice in Science and Technology Education (pp. 89-102). Springer Editorial.

Atadero, R. A., Rambo-Hernandez, K. E., y Balgopal, M. M. (2015). Using social cognitive career theory to assess student outcomes of group design projects in statics. Journal of Engineering Education, 104(1), 55-73.

Baker, T. R., y White, S. H. (2003). The effects of G.I.S. on students' attitudes, Self-efficacy, and achievement in middle school science classrooms. Journal of Geography, 102(6), 243254. https://doi.org/10.1080/00221340308978556

Bandura, A. (2012). On the Functional Properties of Perceived Self-Efficacy Revisited. Journal of Management, 38(1), 9-44.

Bandura, Albert. (1997). Self-efficacy: the exercise of control. New York: W. H. Freeman.

Beier, M. E., Kim, M. H., Saterbak, A., Leautaud, V., Bishnoi, S., y Gilberto, J. M. (2019). The effect of authentic project-based learning on attitudes and career aspirations in STEM. Journal of Research in Science Teacbing, 56(1), 3-23. 
Bong, M., y Skaalvik, E. M. (2003). Academic Self-Concept and Self-Efficacy: How Different Are They Really? Educational Psychology Review, 15(1), 1-40.

Britner, S. L. (2008). Motivation in high school science students: A comparison of gender differences in life, physical, and earth science classes. Journal of Research in Science Teaching, 45(8), 955-970.

Britner, S. L., y Pajares, F. (2006). Sources of science self-efficacy beliefs of middle school students. Journal of Research in Science Teaching, 43(5), 485-499.

Bryant, S. K. (2017). Self-efficacy sources and academic motivation: A qualitative study of 10th graders. Electronic Theses and Dissertations.

Capraro, R. M., \& Corlu, M. S. (2013). Changing views on assessment for STEM projectbased learning. In R. M. Capraro, M. M. Capraro, \& J. R. Morgan (Eds.), STEM ProjectBased Learning an Integrated Science, Technology, Engineering, and Mathematics (STEM) Approach (pp. 109-118). Sense Publishers. https://doi.org/10.1007/978-94-6209-1436_12

Crujeiras Pérez, B., \& Jiménez Aleixandre, M. P. (2018). Influencia de distintas estrategias de andamiaje para promover la participación del alumnado de secundaria en las prácticas científicas. Enseñanza de Las Ciencias, 2(36), 23-42. https://pdfs.semanticscholar.org/399b/8db3e9d6058bb9b1fdeed3fa76bf16fc30cb.pdf

Dewsbury, B., \& Brame, C. J. (2019). Inclusive teaching. CBE Life Sciences Education, 18(2), 1-5. https://doi.org/10.1187/cbe.19-01-0021

Domènech-Casal, J. (2018). Aprendizaje Basado en Proyectos en el marco STEM. Componentes didácticas para la Competencia Científica. Ápice. Revista de Educación Cientifica, 2(2), 29-42.

Fernandez, F. B. (2017). Action research in the physics classroom: the impact of authentic, inquiry based learning or instruction on the learning of thermal physics. Asia-Pacific Science Education, 3(1). https://doi.org/10.1186/s41029-017-0014-z

Ferrés Gurt, C., Marbà Tallada, A., y Sanmartí, N. (2015). Trabajos de indagación de los alumnos: instrumentos de evaluación e identificación de dificultades. Revista Eureka, 12(1), 22-37.

Grimalt-Álvaro, C., y Couso, D. (2018). Toolkit for assessing the promotion of self-efficacy. Part 1, STEAM4U model tools. https://ddd.uab.cat/record/214172

Harrison, H., Birks, M., Franklin, R., \& Mills, J. (2017). Case study research: Foundations and methodological orientations. Forum Qualitative Sozialforschung, 18(1), 1-12. https://doi.org/10.17169/fqs-18.1.2655

Hasni, A., Bousadra, F., Belletête, V., Benabdallah, A., Nicole, M. C., y Dumais, N. (2016). Trends in research on project-based science and technology teaching and learning at K-12 levels: a systematic review. Studies in Science Education, 52(2), 199-231.

Huang, C. (2013). Gender differences in academic self-efficacy: A meta-analysis. European Journal of Psychology of Education, 28(1), 1-35.

Jiménez-Liso, R. (2020). Aprender ciencia escolar implica aprender a buscar pruebas para construir conocimiento (indagación). (D. Couso, R. Jiménez-Liso, C. Refojo, y J. A. Sacristán, Eds.), Enseñando ciencia con ciencia. Madrid: Penguin Random House. 
Ketelhut, D. J. (2007). The impact of student self-efficacy on scientific inquiry skills: An exploratory investigation in river city, a multi-user virtual environment. Journal of Science Education and Technology, 16(1), 99-111. https://doi.org/10.1007/s10956-006-9038-y

Kier, M. W., Blanchard, M. R., Osborne, J. W., y Albert, J. L. (2014). The Development of the STEM Career Interest Survey (STEM-CIS). Research in Science Education, 44(3), 461-481.

Lai, C. L., Hwang, G. J., y Tu, Y. H. (2018). The effects of computer-supported self-regulation in science inquiry on learning outcomes, learning processes, and self-efficacy. Educational Technology Research and Development, 66(4), 863-892. https://doi.org/10.1007/s11423-018-9585-y

Michaels, S., Shouse, A. W., y Schweingruber, H. A. (2007). Learning from Science Investigations. In Ready, Set, SCIENCE! Putting Research to Work in K-8 Science Classrooms (p. 220). The National Academies Press.

Mostafa, T. (2019). Why don't more girls choose to pursue a science career? In PIS A in Focus (Vol. 93, p. 6). Paris: OECD Publishing.

OECD. (2019). PISA 2018 Results (Volume II): Where All Students Can Succeed. OECD Publishing.

Osborne, J. (2014). Teaching Scientific Practices: Meeting the Challenge of Change. Journal of Science Teacher Education, 25, 177-196.

Pajares, F. (2006). Self-efficacy during childhood and adolescence. In Self-efficacy beliefs of adolescents (pp. 339-367).

Rittmayer, A., y Beier, M. (2009). Overview: Self-Efficacy in STEM. Applying Research to Practice Resources, 1-12.

Ro, H. K., y Loya, K. I. (2015). The Effect of Gender and Race Intersectionality on Student Learning Outcomes In Engineering. The Review of Higher Education, 38(3), 359-396.

Somekh, B., y Lewin, C. (Eds.). (2005). Research Methods in the Social Sciences. London: SAGE Publications.

Tanner, K. D. (2013). Structure matters: Twenty-one teaching strategies to promote student engagement and cultivate classroom equity. CBE Life Sciences Education, 12(3), 322-331. https://doi.org/10.1187/cbe.13-06-0115

The GiST project. (2020). Seven principles for a gender-inclusive learning environment. https://www.thegist.edu.au/schools/create-an-inspiring-stem-environment/sevenprinciples-for-a-gender-inclusive-learning-environment/

Thomas, G., Anderson, D., y Nashon, S. (2008). Development of an Instrument Designed to Investigate Elements of Science Students' Metacognition, Self-Efficacy and Learning Processes: The SEMLI-S. International Journal of Science Education, 30(13), 1701-1724.

Thomas, J. W. (2000). A Review of Research on Project-Based Learning.

Tomczak, M., \& Tomczak, E. (2014). The need to report effect size estimates revisited. An overview of some recommended measures of effect size. Trends in Sport Sciences, 1(21), 19-25.

Usher, E. L. (2009). Sources of Middle School Students' Self-Efficacy in Mathematics: A Qualitative Investigation. American Educational Research Journal, 46(1), 275-314.

Webb-Williams, J. (2018). Science Self-Efficacy in the Primary Classroom: Using Mixed Methods to Investigate Sources of Self-Efficacy. Research in Science Education, 48(5), 939-961. 
Anexo 1. Resultados de la comparación entre el nivel inicial de autoeficacia en ciencias entre chicos y chicas para cada centro

Resultados de los contrastes de muestras independientes mediante la prueba $U$ de Mann-Whitney de dos colas entre el nivel inicial de autoeficacia de chicos y chicas para cada centro (NCentroA $=26$ y NCentroB=34).

\begin{tabular}{|c|c|c|c|c|}
\hline \multicolumn{2}{|c|}{ Diferencias iniciales entre chicos y chicas } & $\begin{array}{c}\text { Valor del } \\
\text { estadístico }\end{array}$ & $\begin{array}{l}\text { Valor de la } \\
\text { significancia }\end{array}$ & $\begin{array}{l}\text { Correlación } \\
\text { biserial por }\end{array}$ \\
\hline \multirow{2}{*}{$\begin{array}{l}\text { P1: Diseñar experiencias para tomar } \\
\text { datos }\end{array}$} & Centro A & 80,000 & 1,000 & 0,000 \\
\hline & Centro B & 132,000 & 0,847 & 0,043 \\
\hline \multirow{2}{*}{$\begin{array}{l}\text { P2: Formular hipótesis o } \\
\text { predicciones sobre un experimento }\end{array}$} & Centro A & 81,000 & 0,978 & 0,012 \\
\hline & Centro B & 125,000 & 0,968 & $-0,012$ \\
\hline \multirow{2}{*}{$\begin{array}{l}\text { P3: Identificar qué factores o } \\
\text { condiciones (variables) pueden } \\
\text { afectar a un experimento }\end{array}$} & Centro A & 71,000 & 0,640 & $-0,113$ \\
\hline & Centro B & 150,500 & 0,362 & 0,190 \\
\hline \multirow{2}{*}{$\begin{array}{l}\text { P4: Tomar datos y representarlos } \\
\text { gráficamente }\end{array}$} & Centro A & 55,000 & 0,169 & $-0,313$ \\
\hline & Centro B & 154,500 & 0,294 & 0,221 \\
\hline \multirow{2}{*}{$\begin{array}{l}\text { P5: Dar una explicación científica a } \\
\text { fenómenos o experiencias }\end{array}$} & Centro A & 63,500 & 0,377 & $-0,206$ \\
\hline & Centro B & 111,500 & 0,582 & $-0,119$ \\
\hline \multirow{2}{*}{$\begin{array}{l}\text { P6: Obtener conclusiones a partir de } \\
\text { unos datos tomados }\end{array}$} & Centro A & 46,500 & 0,070 & $-0,419$ \\
\hline & Centro B & 159,500 & 0,206 & 0,261 \\
\hline \multirow{2}{*}{$\begin{array}{l}\text { P7: Argumentar en público los } \\
\text { resultados de una investigación }\end{array}$} & Centro A & 68,000 & 0,524 & $-0,150$ \\
\hline & Centro B & 94,000 & 0,227 & $-0,257$ \\
\hline
\end{tabular}

